\title{
Osteogenesis imperfecta and complications due to pelvic narrowing: case and review
}

\author{
Jakob Bie Granild-Jensen ${ }^{1}$, Line Granild Bie Mertz², Soren Rittig ${ }^{3}$, Gratien Andersen ${ }^{4}$, \\ John Rosendahl Oestergaard ${ }^{5 *}$
}

${ }^{1}$ Department of Paediatrics, Aarhus University Hospital, Skejby, Denmark;

${ }^{2}$ Paediatric Research Laboratory, Aarhus University Hospital, Skejby, Denmark;

${ }^{3}$ Department of Paediatric Nephrology, Aarhus University Hospital, Skejby, Denmark;

${ }^{4}$ Department of Radiology, Aarhus University Hospital, Skejby, Denmark;

${ }^{5}$ Center of Rare Diseases, Aarhus University Hospital, Skejby, Denmark.

Email: *john.oestergaard@skejby.rm.dk

Received 2 October 2011; revised 14 November 2011; accepted 26 November 2011.

\begin{abstract}
We describe a case of pelvic narrowing in a patient with osteogenesis imperfecta (OI) type III and complications consisting of deep venous thrombosis, hydronephrosis and faeculomas. The literature is reviewed and the management is discussed. Conclusion: Monitoring of OI type III patients for pelvic narrowing and complications is recommended.
\end{abstract}

Keywords: Acetabular Protrusion; Bisphosphonate; Constipation; Deep Venous Thrombosis; Hydronephrosis; Pelvis

\section{INTRODUCTION}

Osteogenesis imperfecta (OI) is a rare genetic disorder of type 1 collagen resulting in connective tissue abnormalities. Varying degrees of bone fragility and skeletal deformity are the main characteristics, other important features being dentinogenesis imperfecta and blue sclerae. Based on phenotype and genotype OI is divided into subtypes [1-3].

Pelvic narrowing has been described in a number of disorders of bone metabolism [4]. In OI, pelvic narrowing is probably a consequence of both fractures and bone remodeling. Complications due to pelvic narrowing have been described. Most frequently it is associated with constipation [5].

We report on a girl with OI type III and a triad of gastrointestinal, vascular and urologic complications due to narrowing of the pelvis. The management and outcome is described and the literature is reviewed.

\section{CASE REPORT}

The report concerns a girl born in 1997 in a family with no history of OI. OI was suspected shortly after her birth in 1997 due to congenital femur and rib fractures and blue sclerae. The diagnosis was confirmed by skin biopsy revealing a structural abnormality without quantitative deficiency of type I collagen consistent with OI type III or IV. Exons of all abnormal peptides were sequenced, but no pathogenic mutation was identified. During the first year of life, she had more than 25 fractures. The rate of fractures was reduced by approximately 50\% after repeated bisphosphonate infusions were started (pamidronate 9 - $12 \mathrm{mg} / \mathrm{kg} /$ year from 1998 to 2007, thereafter 4.5 - $6 \mathrm{mg} / \mathrm{kg} /$ year). Furthermore, during the initial 4 years she had telescopic rods placed in the humerus, radius, femur and tibia bilaterally. During 2004-2006 she had few, but recurrent episodes of abdominal pain and constipation despite the use of laxatives. She had no urological complaints or urinary tract infections during her first 10 years of life. In March 2007, she was admitted with left-sided abdominal pain. An ultrasonography disclosed a left hydronephrosis. The pain faded after placement of a nephrostomy tube. Two months later, she was re-admitted, now with severe abdominal pain and constipation. A few weeks earlier, morphine was administered due to fractures and pain. Large faecal masses were palpated in the abdomen. At this time, her left leg became red and swollen and as a thrombosis of the left iliac vein was found by ultrasonography, low molecular weight heparin administration was initiated. CT scan of the pelvic region revealed wedging of the sigmoid colon between the left superior ramus of the pubic bone and the vertebral column. She had bilateral hydronephrosis and hydro-ureter. No calculi were visible. In the sigmoid and transverse colon she had several faeculomas. During operation, it was necessary to do a resection of the sigmoid colon and perform a colostomy. After the operation 
the patient recovered remarkably, and repeated ultrasonography showed improvement of the hydronephrosis on the left side. Kidney function measured as GFR by 51-chrome-EDTA clearance was normal. The severe pelvic narrowing was found due to an almost 90 degree lumbosacral lordosis leaving a space of only $10.3 \mathrm{~mm}$ between the vertebral column and the pubic bones (Figures 1 and 2).

Six months later, a MAG3 renography revealed a great decline in right kidney function (right/left 26\%/ $74 \%$ ) and a cystoscopy showed pelvic bladder compression and dilation of the right ureter and pelvis. Orthope-

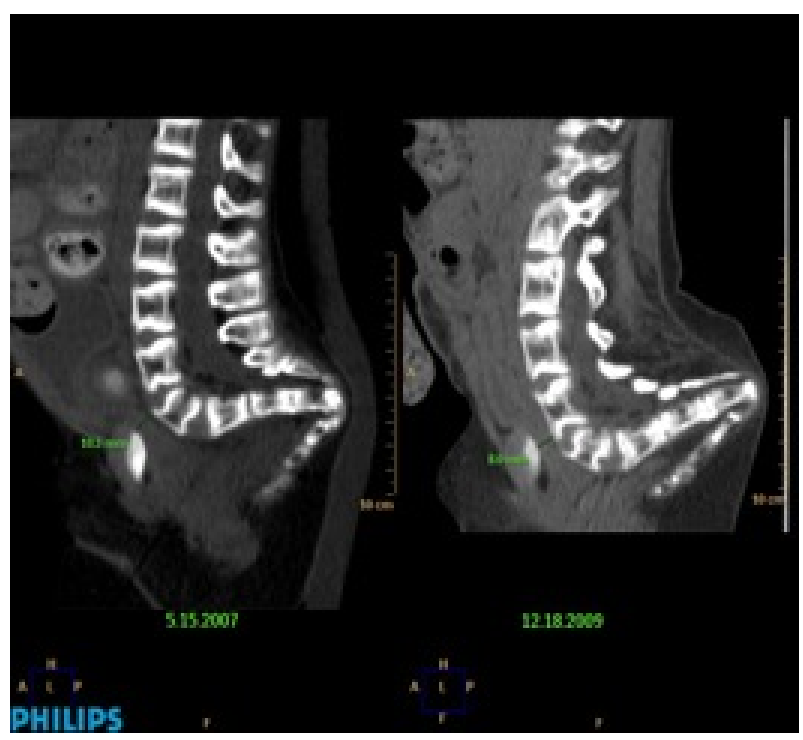

Figure 1. Sagittal multiplanar reconstruction (MPR) of the lumbosacral spine and pelvis.

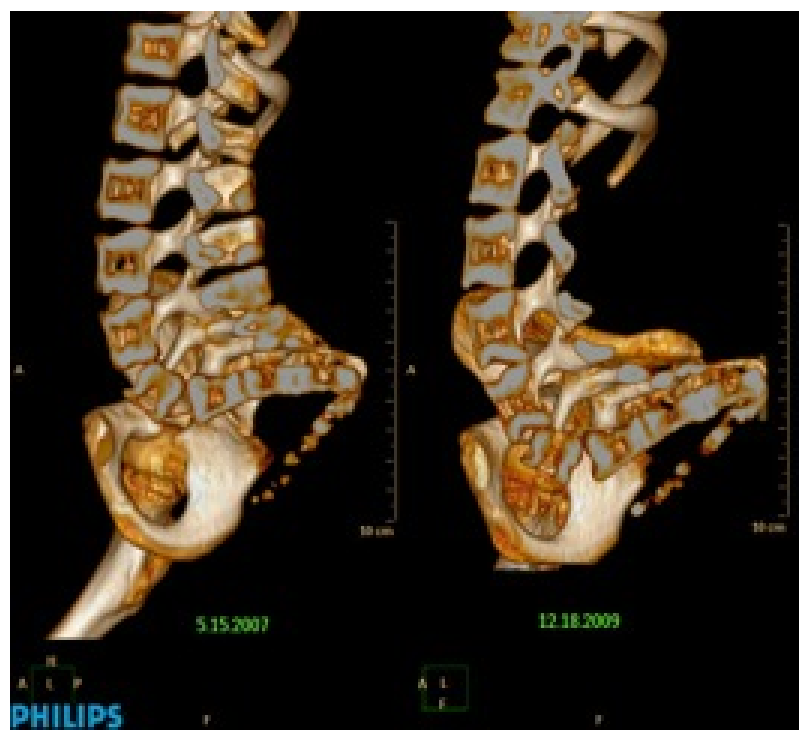

Figure 2. 3D Volume rendering of the lumbosacral spine and pelvis with sagittal cutting plane. dic pelvic reconstruction was considered but the surgical risk taken into account, ureteral reimplantation was prefered. The hydronephrosis improved and the right kidney function improved. One and a half years later, however, the patient suffered several febrile urinary tract infections due to post-voiding residual urine of up to $200 \mathrm{ml}$. A CT scan showed further progression of the pelvic narrowing, the space between the vertebral column and the pubic bones being reduced to $8 \mathrm{~mm}$ (Figures 1 and 2). Prophylactic antibiotic treatment was initiated and a permanent suprapubic catheter was placed. It was later replaced by a continent appendicovesicostomy (Mitrofanoff). During six months following the appendicovesicostomy, she had suffered a single urinary tract infection.

\section{DISCUSSION}

Pelvic deformities in osteogenesis imperfecta are most likely a result of frequent fractures and remodeling of bones. The most studied deformity is acetabular protrusion.

In a study comprising 76 individuals with OI, Violas et al. [5] determined the incidence of acetabular protrusion in OI to 33 per cent. The most frequent complication was constipation.

Wenger et al reported on a 14 year old boy with severe OI and severe intrapelvic acetabular protrusion producing a distal obstruction of the colon [4]. Lee et al. studied 43 patients with type III OI to determine the frequency and severity of abdominal problems and the relationship between these problems and pelvic deformity. The study concluded that chronic constipation and recurrent abdominal pain were more frequent in patients who had acetabular protrusion than in those who did not [6].

Reported renal complications of OI are nephrolithiasis and nephrocalcinosis. These complications can be caused by urinary tract obstruction, urinary stasis caused by scoliosis [7] and hypercalciuria, the latter occurring in one-third of OI patients [8]. Vetter et al. reported on 58 children with various forms of OI. Four of these patients had kidney stones and renal papillary calcifications [9]. In 1995, Butani et al. described chronic renal failure attributed to obstructive uropathy secondary to bony pelvic outlet deformities in a 16 year old boy with OI type III. The authors concluded that the renal failure was caused by the pelvic deformity [7].

The present case illustrates three types of complications attributable to pelvic narrowing in OI. Narrowing of the pelvis is usually the result of acetabular protrusion, and is found most frequently in OI type III, where it occurs in up to two-thirds of the patients [5]. Our patient had only minimal acetabular protrusion. Instead, the 
severe pelvic narrowing was a result of excessive lumbar lordosis and a very short distance between the pubic arch and the lumbar spine. To our knowledge this type of pelvic narrowing has not previously been described in patients with OI. As our patient was treated with bisphophonates for approximately 10 years it raises the question whether this treatment protects from acetabular protrusion, but predisposes for this form of pelvic narrowing.

The initiating factor resulting in the complete triad of faeculomas, deep veinous thrombosis and hydronephrosis seemed to be the development of constipation most probably due to pelvic narrowing. Opioid treatment may have been a contributing factor. It seems rational to improve the reduced pelvic entry by perfoming a pelvic reconstruction as described by Wenger et al. [4]. The surgical morbidity and mortality, however, is considerable and the present case describes how the complications of pelvic narrowing were treated by less invasive methods. The procedures employed were colostomy after resection of the sigmoid colon and ureteral reimplantation. Abdominal pain and defaecation difficulty improved significantly, as did the hydronephrosis and since the colostomy was performed in May 2007, she had had no vascular complications. Post-voiding residual urine and recurrent urinary tract infections were successfully treated with a continent cutaneous appendicovesicostomy.

\section{CONCLUSION}

This case illustrates the need to closely monitor OI patients for pelvic narrowing and for development of faeculomas, deep veinous thrombosis and obstructive nephropathy. It is recommended to prescribe opioid treatment with great care and prophylactic laxatives should be considered. If constipation develops it should be treated promptly. It is advised to keep in mind that pelvic narrowing is not necessarily a result of acetabular protrusion and to note a possible correlation with bisphosphonate treatment.

\section{ACKNOWLEDGEMENTS}

Y.F. Rawashdeh, M.D., Department of Urology, Aarhus University Hospital, Skejby.

I. Hvid, M.D., Prof., Dr. Med., Department of Orthopaedic Surgery, Aarhus University Hospital, Aarhus.

\section{REFERENCES}

[1] Sillence, D.O. and Rimoin, D.L. (1978) Classification of osteogenesis imperfect. Lancet, 1, 1041-1042. doi:10.1016/S0140-6736(78)90763-8

[2] Kocher, M.S. and Shapiro, F. (1998) Osteogenesis imperfecta. Journal of the American Academy of Orthopaedic Surgeons, 6, 225-236.

[3] Shapiro, J.R. and Sponsellor, P.D. (2009) Osteogenesis imperfecta: Questions and answers. Current Opinion in Pediatrics, 21, 709-716. doi:10.1097/MOP.0b013e328332c68f

[4] Wenger, D.R., Abrams, R.A., Yaru, N., et al. (1988) Obstruction of the colon due to protrusio acetabuli in osteogenesis imperfecta: Treatment by pelvic osteotomy: Report of a case. Journal of Bone and Joint Surgery. American Volume, 70, 1103-1107.

[5] Violas, P., Fassier, F., Hamdy, R., et al. (2002) Acetabular protrusion in osteogenesis imperfecta. Journal of Pediatric Orthopaedics, 22, 622-625. doi:10.1097/01241398-200209000-00010

[6] Lee, J.H., Gamble, J.G., Moore, R.E., et al. (1995) Gastrointestinal problems in patients who have type-III osteogenesis imperfecta. Journal of Bone and Joint Surgery. American Volume, 77, 1352-1356.

[7] Butani, L., Rosekrans, J.A., Morgenstern, B.Z., et al. (1995) An unusual renal complication in a patient with osteogenesis imperfecta. American Journal of Kidney Diseases, 25, 489-491. doi:10.1016/0272-6386(95)90114-0

[8] Chines, A., Petersen, D.J., Schranck, F.W., et al. (1991) Hypercalciuria in children severely affected with osteogenesis imperfecta. The Journal of Pediatrics, 119, 51-57. doi:10.1016/S0022-3476(05)81038-8

[9] Vetter, U., Maierhofer, B., Muller, M., et al. (1989) Osteogenesis imperfecta in childhood: Cardiac and renal manifestations. European Journal of Pediatrics, 149, 184-187. doi:10.1007/BF01958277 